\title{
EDITORIAL:
}

\section{SOFTWARE SURVEY SECTION}

The introduction of the new Software Survey Section to INSECT SCIENCE AND ITS APPLICATION is to encourage the open exchange of information on software programmes unique to our professional field. With the rapid penetration of computers into academic and industrial institutions has come a parallel increase in the number of scientists and researchers designing their own software. The existence of much of this software remains unknown even to those of us who could most benefit from its use. We believe that it is of vital importance to our readers that such information be made available. We also believe that a professional journal is the best place to share such information. Your contribution would be most welcome. 